filing applied to primary hepatoctyes and hepatocyte cell lines Hep-G2 and Hep-3B.J Proteome Res. 2010;9(1):6-21

17. Matsuo N, et al. Twist expression promotes migration and invasion in hepatocellular carcinoma. BMC Cancer. 2009;9:240.

18. Sicklick JK, et al. Dysregulation of the Hedgehog pathway in human hepatocarcinogenesis. Carcinogenesis. 2006;27(4):748-757.

19. Katoh Y, Katoh M. Hedgehog signaling, epithelialto-mesenchymal transition and miRNA (review). Int J Mol Med. 2008;22(3):271-275.

20. Katoh Y, Katoh M. Hedgehog target genes: mechanisms of carcinogenesis induced by aberrant hedgehog signaling activation. Curr Mol Med. 2009;9(7):873-886

21. Fuchs BC, et al. Epithelial-to-mesenchymal transition and integrin-linked kinase mediate sensitivity to epidermal growth factor receptor inhibition in human hepatoma cells. Cancer Res. 2008;68(7):2391-2399.

22. Lahsnig C, et al. ILE1 requires oncogenic Ras for the epithelial to mesecnhymal transition of hepatocytes and liver carcinoma progression. Oncogene. 2009;28(5):638-650.

23. Parola M, Pinzani M. Hepatic wound repair. Fibrogenesis Tissue Repair. 2009;2(1):4.

24. Wells RG. The epithelial-to-mesenchymal transition in liver fibrosis: here today, gone tomorrow? [published online ahead of print January 27, 2010]. Hepatology. doi:10.1002/hep.23529.

25. Choi SS, Diehl AM. Epithelial-to-mesenchymal transitions in the liver. Hepatology. 2009;50(6):2007-2013.

26. Sackett SD, et al. Foxl1 is a marker of bipotential hepatic progenitor cells in mice. Hepatology.
2009;49(3):920-929.

27. Taura K, et al. Hepatocytes do not undergo epithelial-mesenchymal transition in liver fibrosis in mice [published online ahead of print October 13, 2009]. Hepatology. doi:10.1002/hep.23368.

28. Witek RP, et al. Liver cell-derived microparticles activate hedgehog signaling and alter gene expression in hepatic endothelial cells. Gastroenterology. 2009; 136:320-330.

29. Min AL, et al. High expression of Snail mRNA in blood from hepatocellular carcinoma patients with extra-hepatic metastasis. Clin Exp Metastasis. 2009; 26(7):759-767.

30. Murata K, Suzuki H, Okano H, Oyamada T, Yasuda T, Sakamoto A. Hypoxia-induced des-gamma-carboxy prothrombin production in hepatocellular carcinoma. Int J Oncol. 2010;36(1):161-170.

\title{
Monkeying around with cardiac progenitors: hope for the future
}

\author{
Li Qian and Deepak Srivastava \\ Gladstone Institute of Cardiovascular Disease, Department of Pediatrics, and \\ Department of Biochemistry and Biophysics, University of California, San Francisco, California
}

\begin{abstract}
Multipotent cardiovascular progenitor cells derived from ES cells or induced pluripotent stem (iPS) cells are an intriguing source for stem cellbased therapies for congenital and acquired heart diseases. From a clinical perspective, the ideal cardiac progenitor cells are those that can proliferate, survive, and differentiate into multiple mature cardiac cell types when transplanted into normal or diseased heart. In this issue of JCI, Blin et al. report the isolation and characterization of a group of early mesodermal cardiovascular progenitor cells, induced by BMP2 and marked by the cell surface protein, stage-specific embryonic antigen 1 (SSEA-1). BMP2induced SSEA-1 ${ }^{+}$cells were purified from ES and iPS cells and could be directed to differentiate into cardiomyocytes, endothelial cells, and smooth muscle cells by treatment with defined cytokines and signaling molecules. Most importantly, purified SSEA ${ }^{+}$progenitor cells from Rhesus monkey ES cells engrafted into nonhuman primate hearts, in which they differentiated into cardiac cells without forming teratomas. These findings move the field another step closer to clinical use of ES or iPS cell-derived cardiovascular progenitors in cardiac repair.
\end{abstract}

Heart failure is a progressive disease that affects over 5 million individuals in the United States. In addition, many of the over 1 million survivors of congenital heart disease are destined to develop heart failure as they age. Current pharmacologic therapies have limited efficacy and only slow the progression of cardiac dysfunction. Heart transplantation is often the last resort of treatment, but the limited donor pool makes this option unrealistic for the

Conflict of interest: Deepak Srivastava serves on the Scientific Advisory Board of iPierian Inc. and RegeneRx Pharmaceuticals.

Citation for this article: J Clin Invest. 2010; 120(4):1034-1036. doi:10.1172/JCI42643. vast majority of patients. Given the disease burden and the unsatisfying therapeutic modalities for heart failure, the potential of cardiac regenerative medicine has generated tremendous interest worldwide.

Approaches to regenerate functional myocardium in damaged hearts have made substantial strides in recent years. Of major tiple cardiovascular progenitor cells (CPCs) that have embryonic origin and can be isolated from heart tissue, or that can be differentiated from ES or induced pluripotent stem (iPS) cells (1-4). The previously characterized CPCs retain intrinsic competence to differentiate into various cardiac lineages. Among them, $\mathrm{Flk}^{+}\left(\mathrm{KDR}^{+}\right.$in human) preimportance was the identification of mul- cursors retain the capacity to differentiate into blood cells and three of the major cell types of the heart cells, namely cardiomyocytes, smooth muscle cells, and endothelial cells $(5,6)$. CPCs expressing the transcription factor Isl1 are also multipotent and give rise to cardiomyocytes, smooth muscle cells, and endothelial cells (7-9). Finally, CPCs marked by the transcription factor $\mathrm{Nkx} 2.5$ are more lineage restricted but can differentiate into cardiomyocytes and smooth muscle cells (10). Despite these advances, practical isolation of cardiac lineage-committed progenitor cells using a surface marker and introduction of such cells into a damaged heart has remained problematic.

\section{Isolation of cardiovascular progenitors using a cell-surface marker}

In this issue of the JCI, Blin and colleagues identified a very early cardiac progenitor population derived from primate (human and monkey) ES and iPS cells. They used knowledge from embryonic development and mimicked the conditions that may be present in the early primitive streak, just as epiblasts give rise to newly formed mesodermal cells that are destined to acquire a cardiac fate (Figure 1). Such cells express transient but high levels of the transcription factors Oct4 and Mesp1 (11). Blin et al. treated ES and iPS cells with BMP2, which activates Wnt3a, to simulate early epiblast conditions, and then used magnetic 


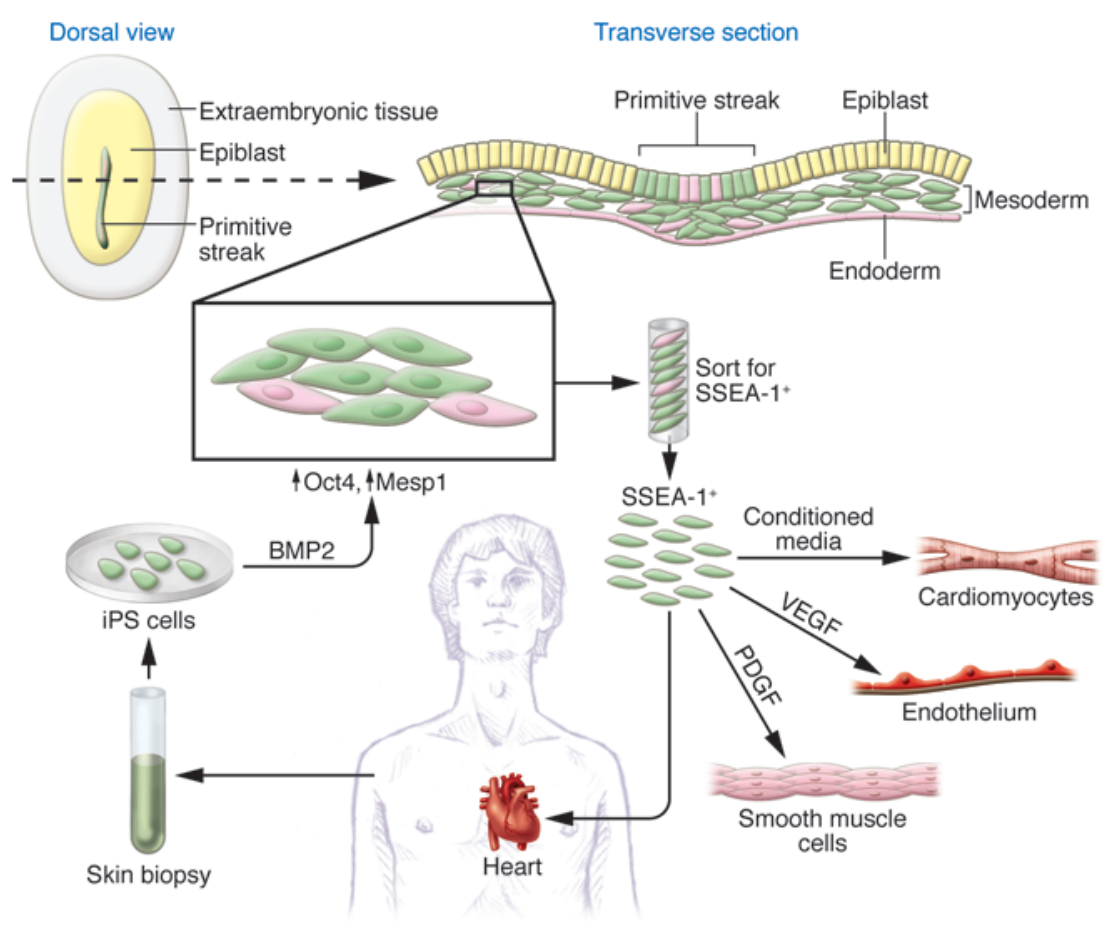

Figure 1

Schematic of primate BMP2-induced SSEA-1+ cells. Epiblast cells in the embryo express the surface marker SSEA-1 as they differentiate and transiently express Oct4 and Mesp1 during the commitment to CPCs. Blin et al. show that using BMP2 to mimic the environment of epiblast cells, primate ES and iPS cells can be induced to differentiate into SSEA-1+ CPCs. Isolated BMP2-induced SSEA-1 + cells were multipotent CPCs that could be differentiated into endothelial cells, smooth muscle cells, and cardiomyocytes under different growth conditions. The SSEA-1+ cells were also multipotent in vivo upon transplantation into primate hearts.

beads to isolate cells that expressed stagespecific embryonic antigen 1 (SSEA-1), an early surface marker of general differentiation. Remarkably, the isolated SSEA-1 $1^{+}$ cells expressed early mesodermal and cardiac lineage markers and appeared by gene expression and epigenetic marks to be very early CPCs.

Only a small portion of SSEA- $1^{+}$CPCs were Flk $1^{+}(\sim 5 \%-7 \%)$, reflecting a difference in developmental stage between the two CPC populations and suggesting the SSEA- $1^{+}$ CPCs might appear earlier than Flk $1^{+}$and other previously isolated CPCs. Of particular interest, BMP2-induced SSEA- $1^{+}$CPCs exhibited an interesting pattern of microRNA expression. Specifically, the miR-17-92 cluster (e.g., miR-106, miR-513, and miR302a) was upregulated, while miR-302b,c,d and miR-125b were downregulated. Future studies of SSEA- $1^{+}$CPC-specific microRNAs (e.g., miR-513) will help to elucidate the biological mechanisms underlying the ability to differentiate toward a cardiogenic fate.

To demonstrate the multipotency and clonality of the SSEA- $1^{+}$CPCs quantita- tively and qualitatively, Blin et al. elegantly used high-content cell imaging and flow cytometry (11). The cells could be induced to differentiate into any one of the three major cardiac lineages, simply by changing the conditions in which they were maintained (Figure 1). For example, when treated with PDGF or VEGF, the CPCs developed into smooth muscle or endothelial cells, respectively. In the presence of conditioned medium of both fibroblasts and cardiomyocytes, they differentiated mostly into ventricular-like cardiomyocytes. The ability to manipulate the fate of SSEA- $1^{+}$CPCs will be important for generating large numbers of relatively pure cells for clinically relevant testing or basic biology studies.

\section{Implications for regenerative medicine}

Powered in part by the discovery of iPS cells (12-15), CPCs or cardiomyocytes derived from pluripotent cells have provided a potential avenue for cardiac regenerative approaches. In particular, the plasticity and cardiogenic differentiation potential of SSEA- $1^{+}$CPCs make them attractive sources for cells to replace damaged myocytes in an infarcted heart.

A true test of the value of these cells is to determine whether they functionally incorporate themselves into heart tissue. Before moving to animal graft experiments, Blin and colleagues examined the maturation of cardiomyocytes derived from BMP2induced SSEA- $1^{+}$CPCs ex vivo (11). A large proportion of differentiated cells $(60 \%-$ $80 \%$ ) displayed organized sarcomeres and expressed cardiac $\alpha$-actinin, ventricular myosin light chain, and the adult $\beta$-myosin heavy chain isoform. Importantly, the gap junction protein connexin 43 (Cx43) was phosphorylated in these cells, indicating the potential for cell-cell coupling. However, whether these cardiomyocytes are in fact electrophysiologically functional will have to be established by standard patch-clamp single-cell recordings.

As a proof of concept, Blin and colleagues transplanted Rhesus ES cell-derived SSEA-1 ${ }^{+}$ CPCs into a Rhesus monkey model of myocardial infarction (11). Previous studies used human ESC-derived cardiomyocytes or CPCs in a rodent myocardial infarction model $(16,17)$, but the human cardiomyocytes failed to couple to rodent host myocardium, possibly due to differences in their intrinsic beating frequency. The unsuccessful coupling might explain the absence of long-term (i.e., up to 12 weeks) functional benefits (16). The Rhesus SSEA- $1^{+}$CPCs engrafted in the infarcted monkey hearts, without forming teratomas, and differentiated into morphologically matured cardiomyocytes that were positive for myosin light chain 2 and myosin light chain kinase.

However, a major goal of cardiac repair is to restore long-term function in an effort to prevent or treat heart failure. Newly formed muscle must provide passive mechanical support and, more importantly, couple and contract in synchrony with the host myocardium. Using immunohistochemistry, Blin et al. reported activation of $\mathrm{Cx} 43$ expression within and surrounding the graft. Although this indicates electrical coupling between the graft and the surrounding myocardium, additional electrophysiological studies (e.g., intracellular calcium imaging) will be needed to unequivocally show successful coupling. Since incomplete coupling can cause ectopic electrical activity and increase the risk of arrhythmia, more functional analyses are needed to prove the procedure is safe and effective. Moreover, the functional benefit of cells 
engrafted into nonhuman primate hearts remains to be determined.

Despite these issues, the pioneering studies of Blin et al. are very encouraging. Their imaginative work using a nonhuman primate model of myocardial infarction to test the capacity of SSEA- $1^{+}$CPCs to engraft and differentiate into matured cardiomyocytes represents an important milestone. Future studies on the long-term survival, functional integration, physiological compatibility of engrafted cells, and beneficial effects on cardiac function will provide new insights into the potential use of SSEA- $1^{+}$CPCs for cardiovascular regenerative medicine. Most importantly, the ability to isolate nonhuman primate CPCs using a cell surface marker brings us one step closer to the ultimate dream of cell-based therapies for some of the most devastating forms of heart disease.

Address correspondence to: Deepak Srivastava, Gladstone Institute of Cardiovascular Disease, University of California, 1650 Owens Street, San Francisco, CA 94158.
Phone: 415.734.2716; Fax: 415.355.0141; E-mail: dsrivastava@gladstone.ucsf.edu.

1. Murry CE, Keller G. Differentiation of embryonic stem cells to clinically relevant populations: lessons from embryonic development. Cell. 2008;132(4):661-680.

2. Srivastava D, Ivey KN. Potential of stem-cellbased therapies for heart disease. Nature. 2006; 441(7097):1097-1099.

3. Passier R, van Laake LW, Mummery CL. Stem-cellbased therapy and lessons from the heart. Nature. 2008; 453(7193):322-329

4. Yi BA, Wernet O, Chien KR. Pregenerative medicine: developmental paradigms in the biology of cardiovascular regeneration. J Clin Invest. 2010; 120(1):20-28.

5. Kattman SJ,Huber TL, Keller GM. Multipotent flk-1+ cardiovascular progenitor cells give rise to the cardiomyocyte, endothelial, and vascular smooth muscle lineages. Dev Cell. 2006;11(5):723-732.

6 . Yang L, et al. Human cardiovascular progenitor cells develop from a $\mathrm{KDR}+$ embryonic-stem-cell-derived population. Nature. 2008;453(7194):524-528.

7. Bu L, et al. Human ISL1 heart progenitors generate diverse multipotent cardiovascular cell lineages. Nature. 2009;460(7251):113-117.

8. Laugwitz KL, et al. Postnatal isl1+ cardioblasts enter fully differentiated cardiomyocyte lineages. Nature. 2005;433(7026):647-653.

9. Moretti A, et al. Multipotent embryonic isl1+ progenitor cells lead to cardiac, smooth muscle, and endothelial cell diversification. Cell. 2006; 127(6):1151-1165.

10. Wu SM, et al. Developmental origin of a bipotential myocardial and smooth muscle cell precursor in the mammalian heart. Cell. 2006;127(6):1137-1150.

11. Blin G, et al. A purified population of multipotent cardiovascular progenitors derived from primate pluripotent stem cells engrafts in postmyocardial infarcted nonhuman primates. $J$ Clin Invest. 2010;120(4):1125-1139.

12. Park IH, et al. Reprogramming of human somatic cells to pluripotency with defined factors. Nature. 2008;451(7175):141-146.

13. Takahashi $\mathrm{K}$, et al. Induction of pluripotent stem cells from adult human fibroblasts by defined factors. Cell. 2007;131(5):861-872.

14. Takahashi K, Yamanaka S. Induction of pluripotent stem cells from mouse embryonic and adult fibroblast cultures by defined factors. Cell. 2006; 126(4):663-676.

15. Yu J, et al. Induced pluripotent stem cell lines derived from human somatic cells. Science. 2007; 318(5858):1917-1920.

16. van Laake LW, et al. Human embryonic stem cellderived cardiomyocytes survive and mature in the mouse heart and transiently improve function after myocardial infarction. Stem Cell Res. 2007; $1(1): 9-24$.

17. Laflamme MA, et al. Cardiomyocytes derived from human embryonic stem cells in pro-survival factors enhance function of infarcted rat hearts. Nat Biotechnol. 2007;25(9):1015-1024.

\title{
Prevention trumps treatment of antibody-mediated transplant rejection
}

\author{
Stuart J. Knechtle, Jean Kwun, and Neal Iwakoshi
}

Emory University School of Medicine, Atlanta, Georgia.

\begin{abstract}
Belying the spectacular success of solid organ transplantation and improvements in immunosuppressive therapy is the reality that long-term graft survival rates remain relatively unchanged, in large part due to chronic and insidious alloantibody-mediated graft injury. Half of heart transplant recipients develop chronic rejection within 10 years - a daunting statistic, particularly for young patients expecting to achieve longevity by enduring the rigors of a transplant. The current immunosuppressive pharmacopeia is relatively ineffective in preventing late alloantibody-associated chronic rejection. In this issue of the JCI, Kelishadi et al. report that preemptive deletion of $B$ cells prior to heart transplantation in cynomolgus monkeys, in addition to conventional posttransplant immunosuppressive therapy with cyclosporine, markedly attenuated not only acute graft rejection but also alloantibody elaboration and chronic graft rejection. The success of this preemptive strike implies a central role for $B$ cells in graft rejection, and this approach may help to delay or prevent chronic rejection after solid organ transplantation.
\end{abstract}

\section{Acute and chronic rejection}

Newly transplanted organs are susceptible within a week to acute rejection, medi-

Conflict of interest: Stuart J. Knechtle acknowledges stock ownership in Bristol-Myers Squibb.

Citation for this article: J Clin Invest. 2010; 120(4):1036-1309. doi:10.1172/JCI42532. ated dominantly by $\mathrm{T}$ cells, but are usually effectively protected from this form of inflammation and injury by currently used immunosuppressive agents such as calcineurin inhibitors, antiproliferative agents, mTOR inhibitors, and prophylactic therapy with $\mathrm{T}$ cell-specific antibodies. When acute rejection occurs, as it does in $5 \%-25 \%$ of solid organ recipients within the first year, it can typically be successfully treated with steroid therapy or, if needed, $\mathrm{T}$ cell-specific antibodies. However, the current immunosuppressive pharmacopeia is relatively ineffective in preventing or treating rejection mediated by $\mathrm{B}$ cells and the antibodies they produce. Antibody-mediated allograft injury, which occurs in $50 \%$ of heart transplant patients within 10 years, typically manifests more than a year after transplantation, more insidiously than $\mathrm{T}$ cell-mediated injury, and in a process characterized by complement deposition and microvascular obliteration that leads to tissue ischemia and eventually fibrosis with loss of graft function. Chronic graft rejection refers to this antibody-mediated process.

While factors contributing to chronic injury of organ transplants are multiple and include ischemia/reperfusion injury, preexisting donor disease, drug toxicities, and recurrence of original disease, the 